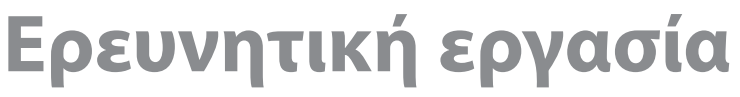 Research article
}

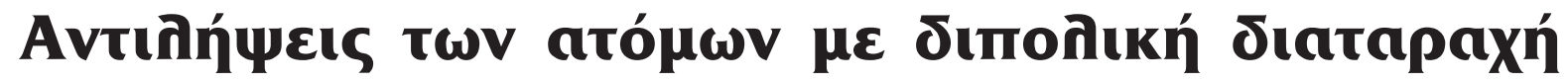

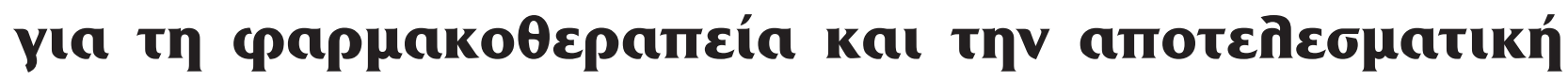

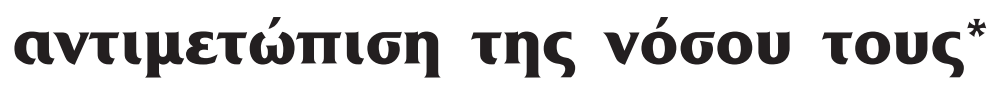

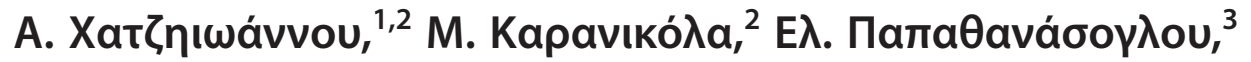

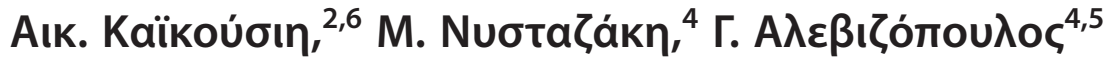

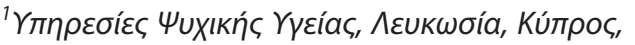

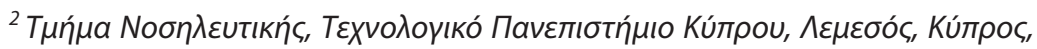

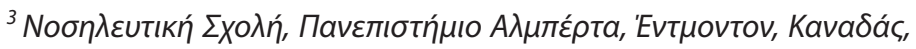

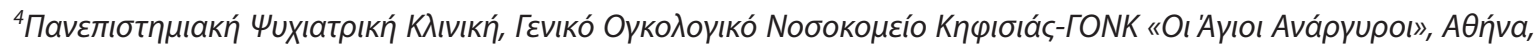

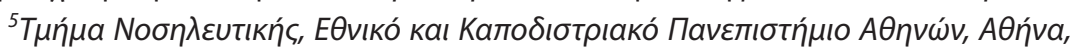

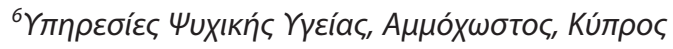 \\ Uuxıatpıkń 2017, 28:291-300
}

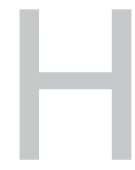

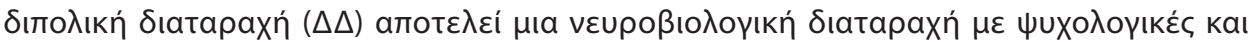

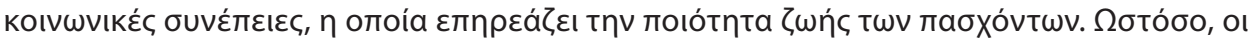

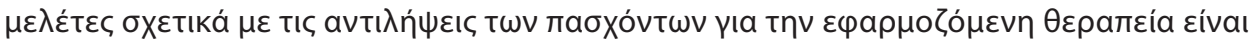

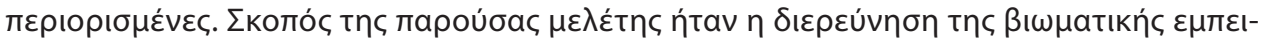

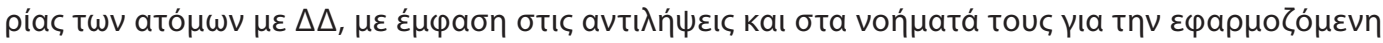

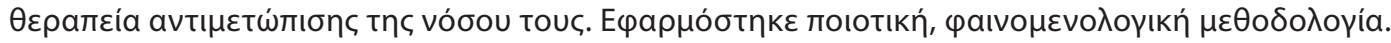

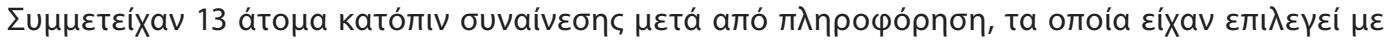

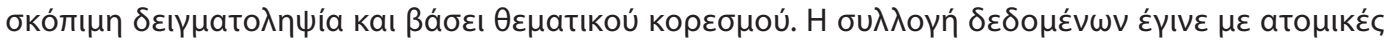

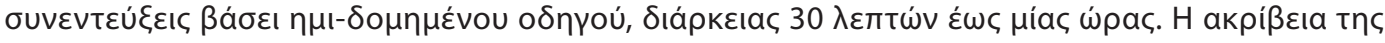

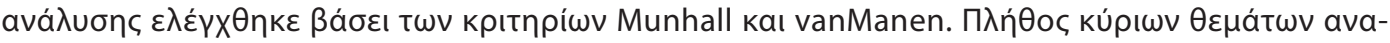

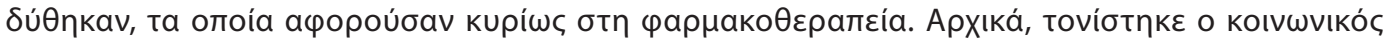

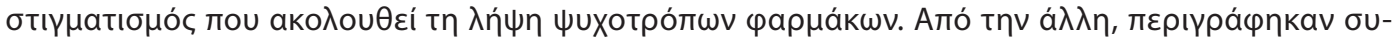

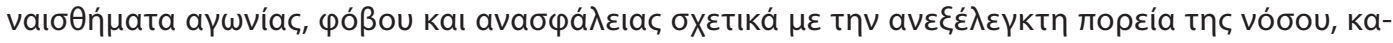

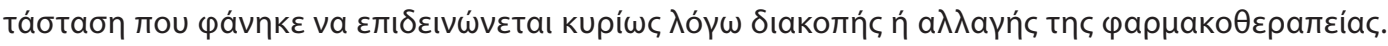

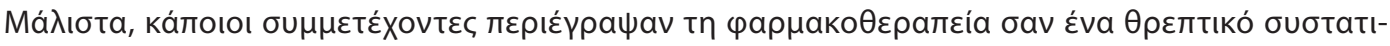

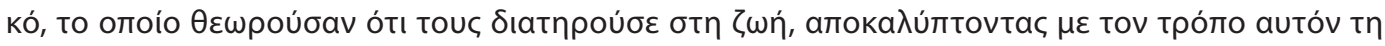

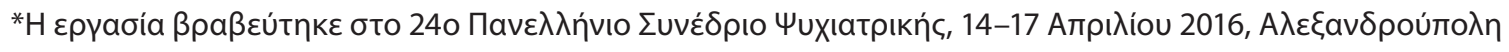




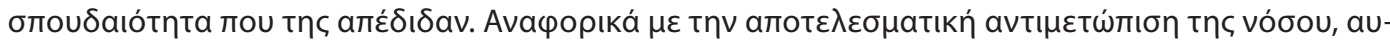

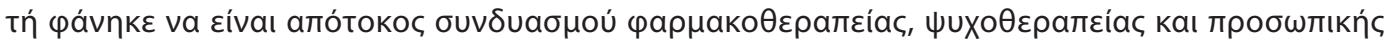

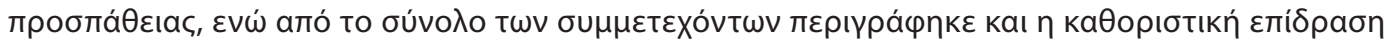

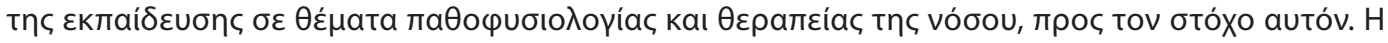

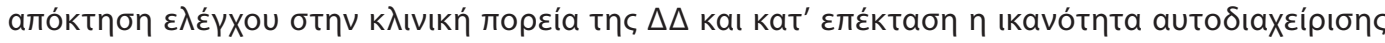

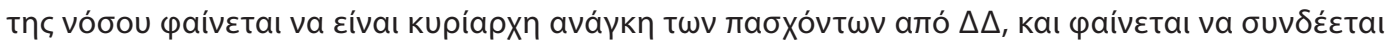

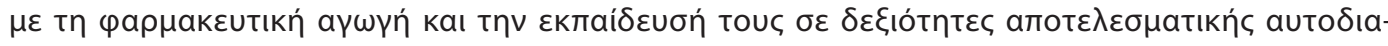

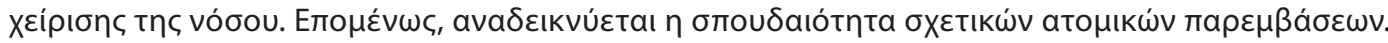

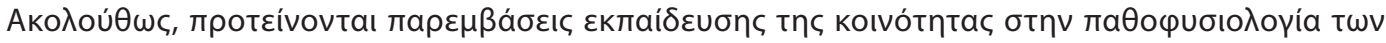

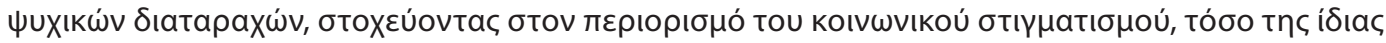

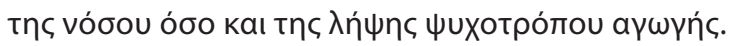

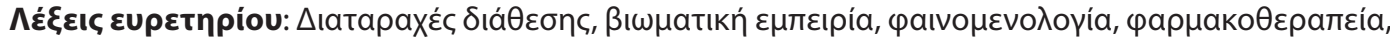

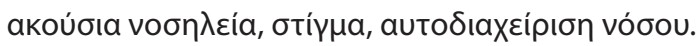

\section{Eıбaywyń}

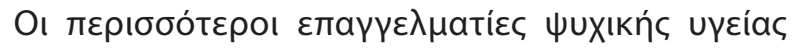

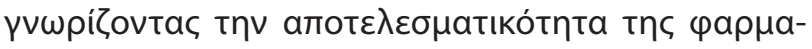

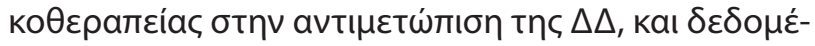

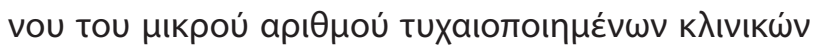

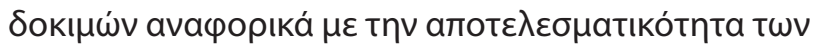

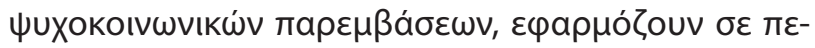

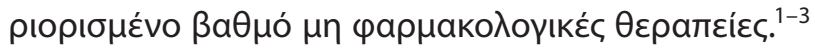

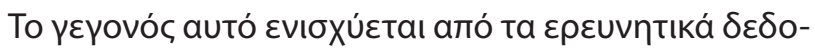

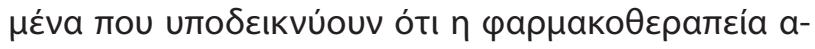

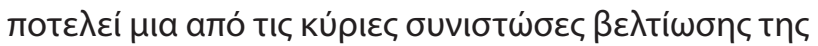

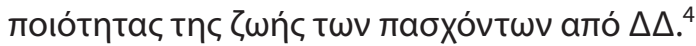

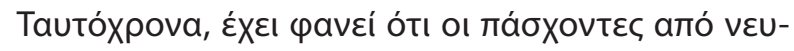

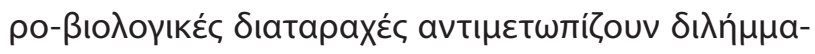

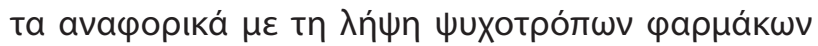

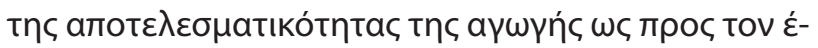

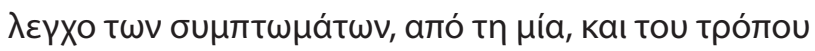

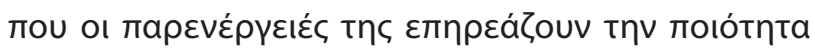

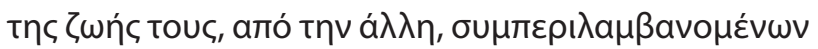

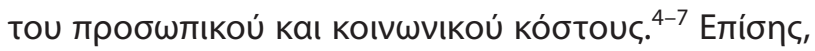

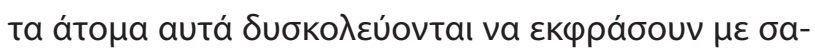

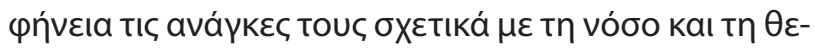

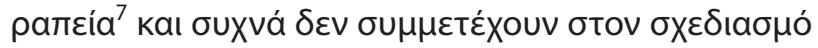

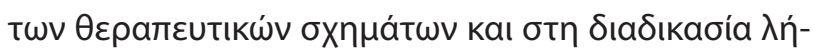

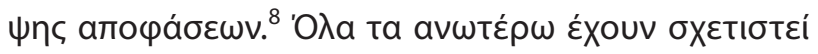

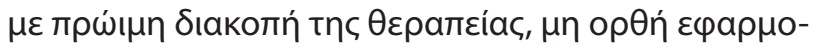
үท́ тпৎ, кaӨ

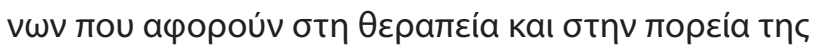

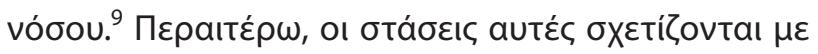

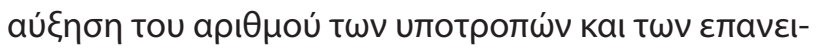

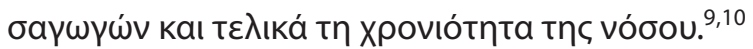

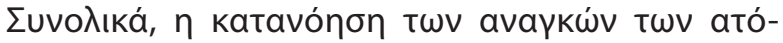

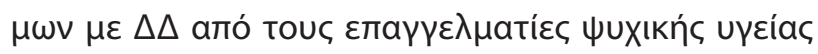

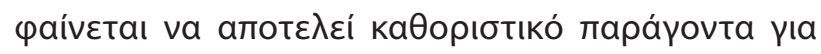

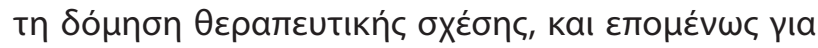

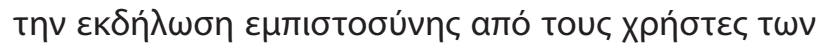

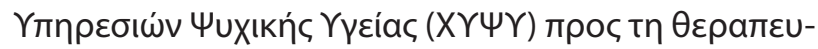

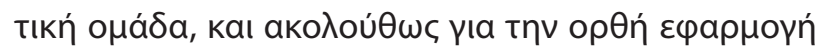

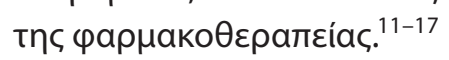

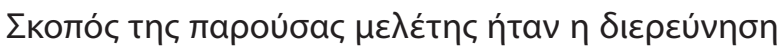

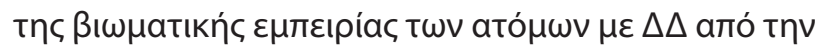

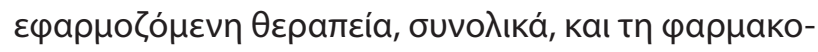

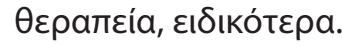

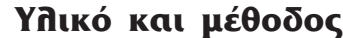

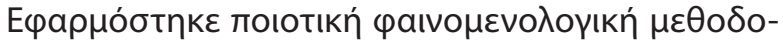

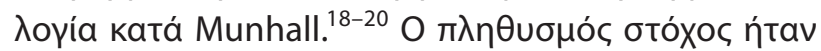

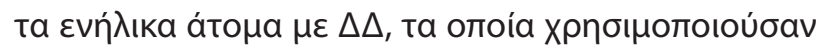

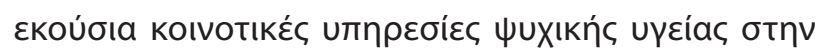

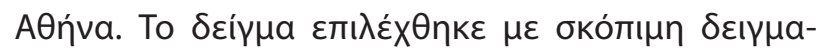

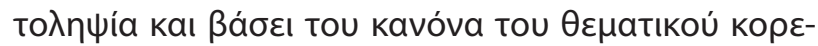

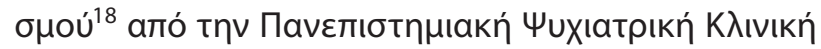

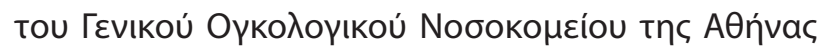

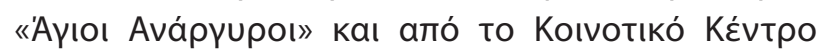

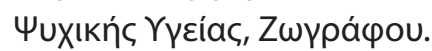

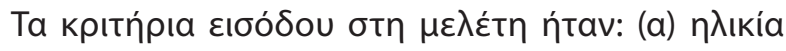

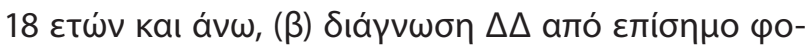

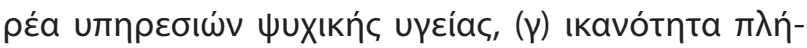

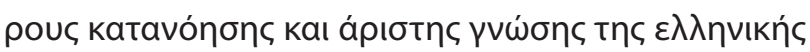

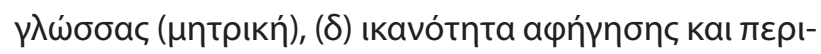

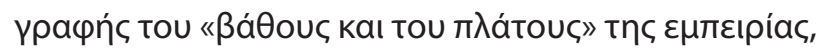

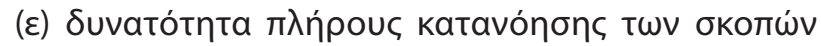

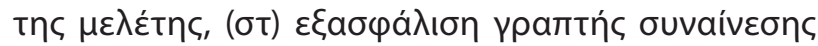




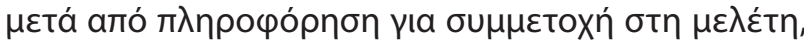

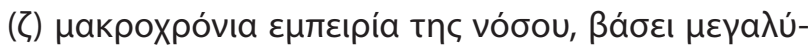

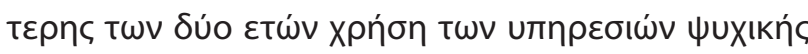

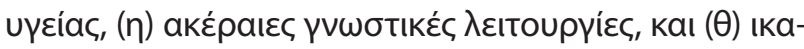

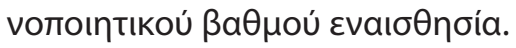

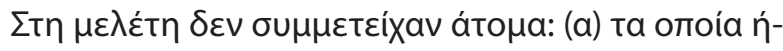

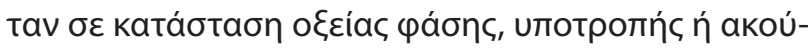

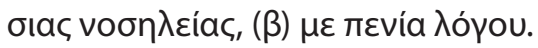

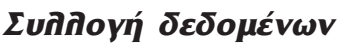

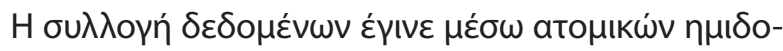

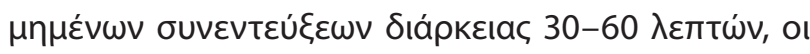

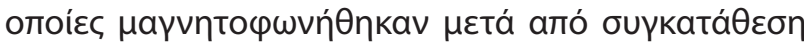

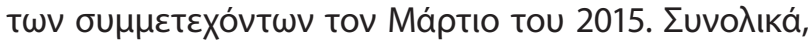

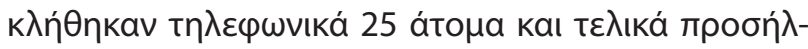

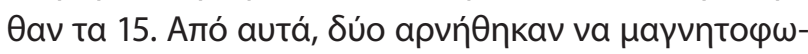

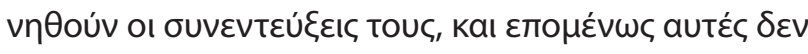

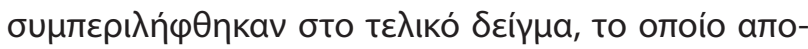

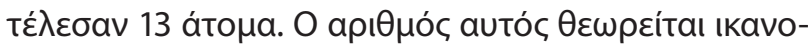

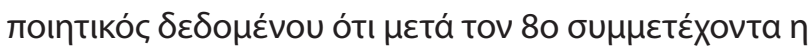

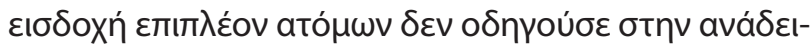

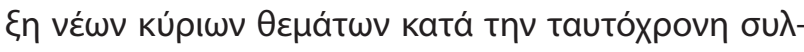

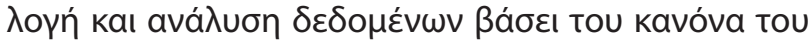

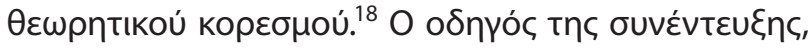

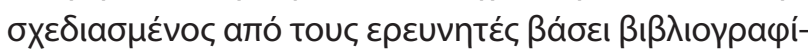

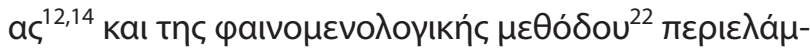

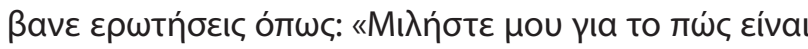

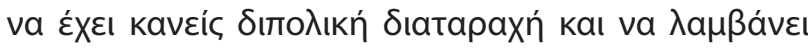

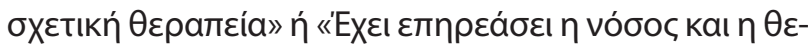

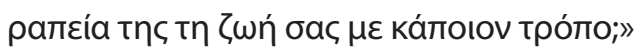

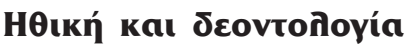

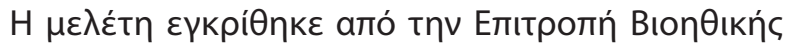

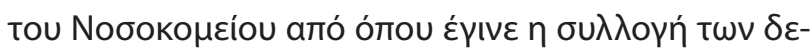

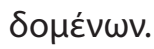

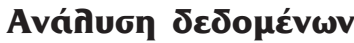

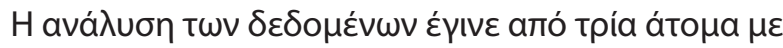

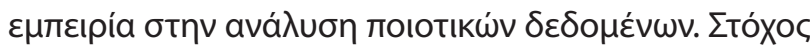

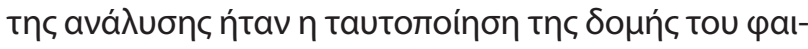

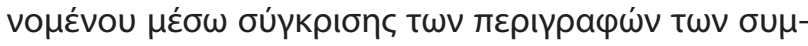

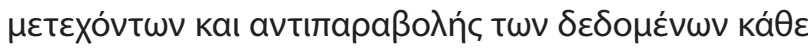

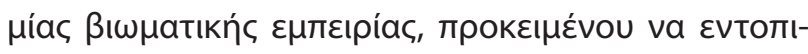

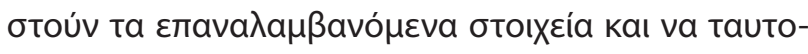

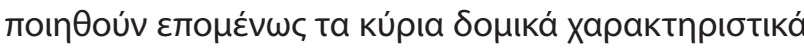

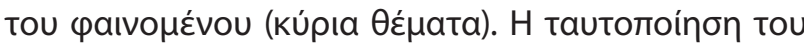

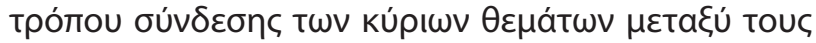

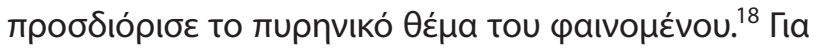

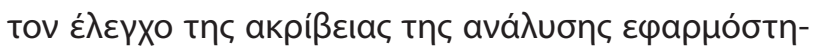
kav ta kpıtńpıa katá van Manen ${ }^{20,21}$ kaı Munhall. ${ }^{18}$

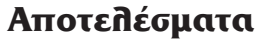

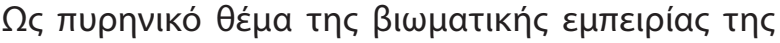

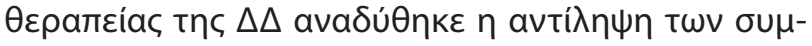

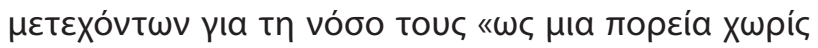

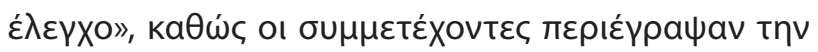

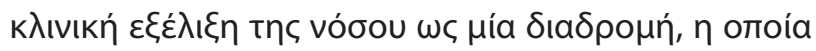

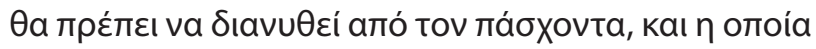

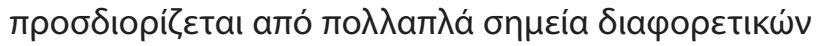

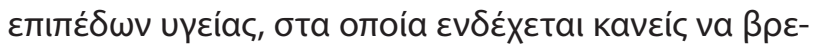

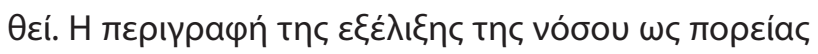

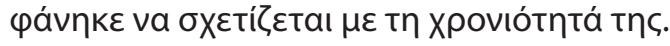

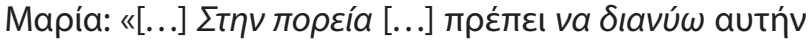

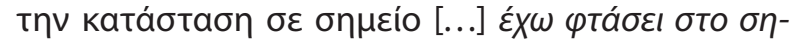
$\mu \varepsilon i ́ o[. .]$.$» .$

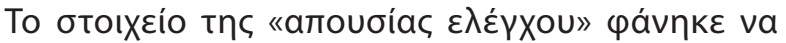

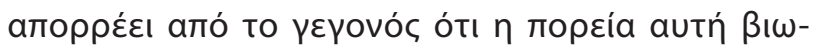

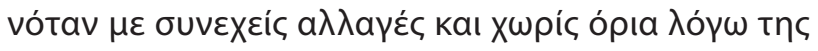

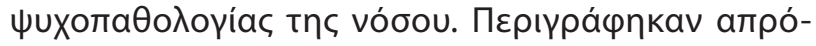

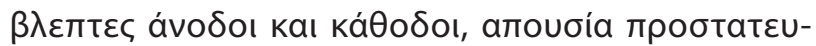

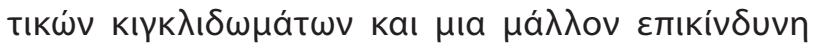

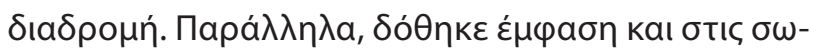

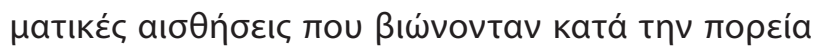

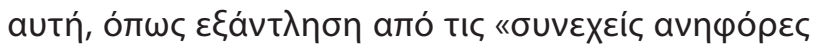

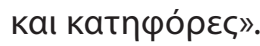

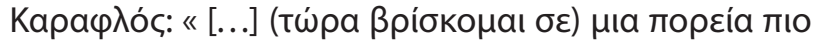

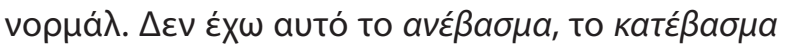
$[\ldots] \gg$.

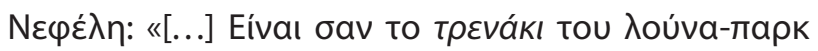

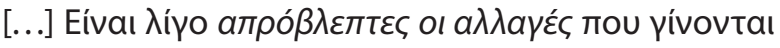

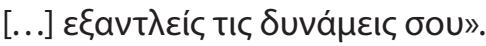

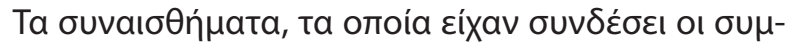

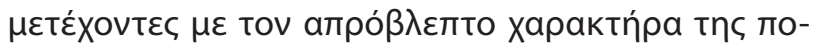

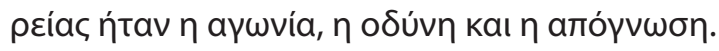

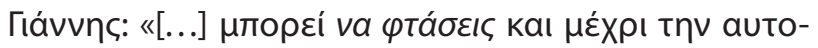
KTovía [...]».

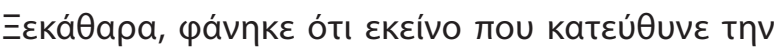

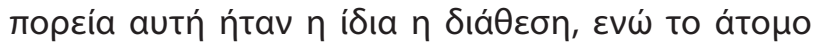

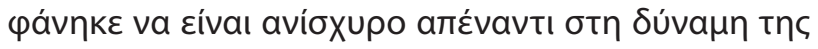

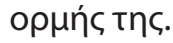




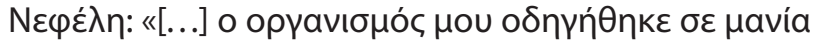

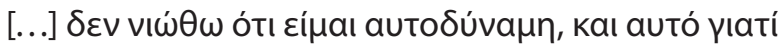

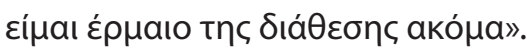

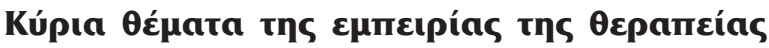 tns $\Delta$ เтоהাкńs $\Delta$ เatapaxńs.}

\section{Н фарнакоөврапві́⿱}

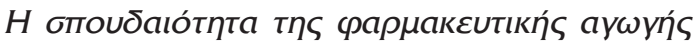

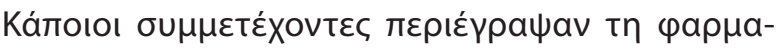

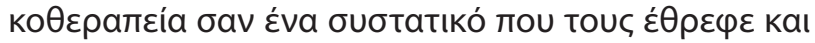

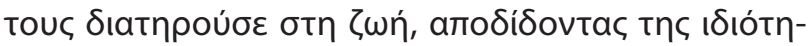

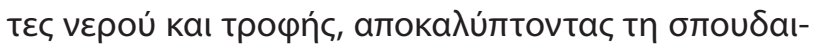

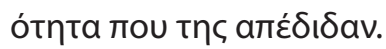

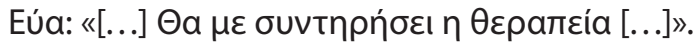

Кámoıı á $\lambda \lambda$ o

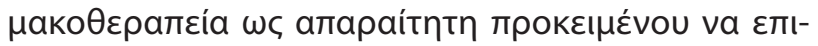

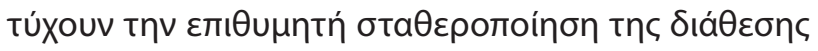

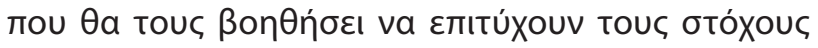

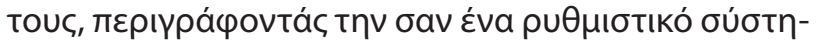
$\mu$.

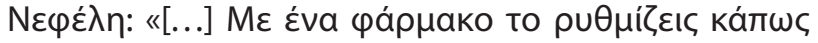

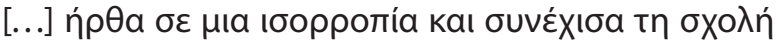
$\mu \mathrm{OU}[\ldots] »$.

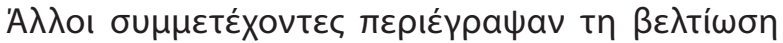

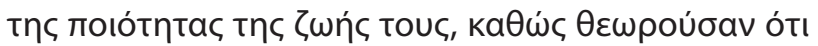

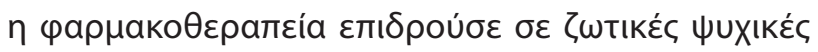

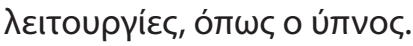

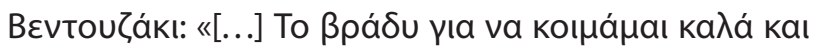

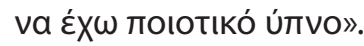

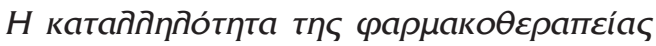

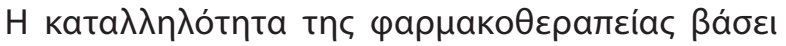

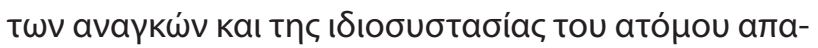

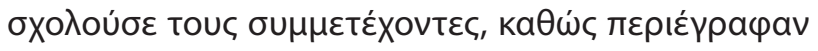

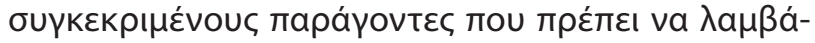

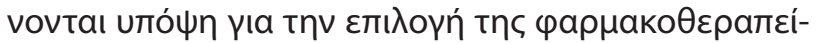

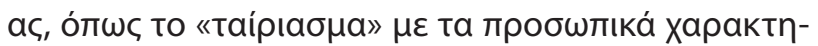
pıбтікá.

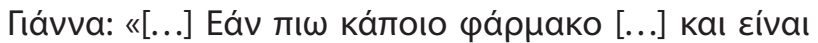

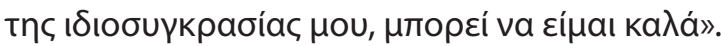

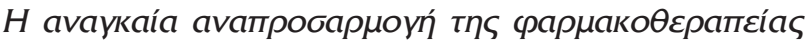

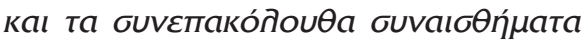

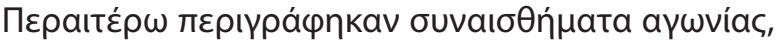

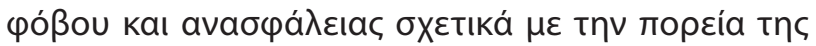

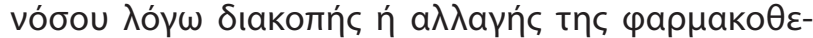

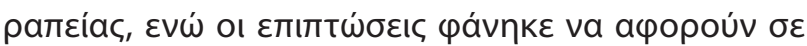

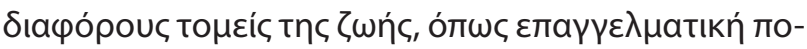

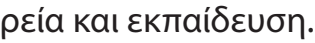

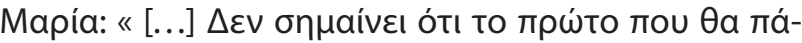

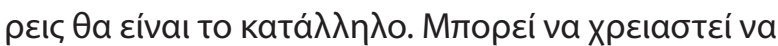

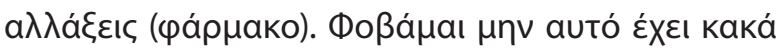

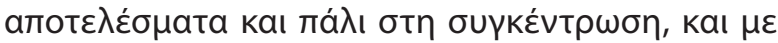

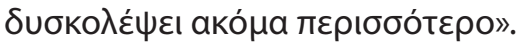

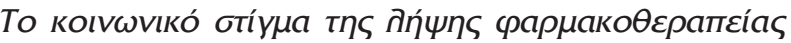

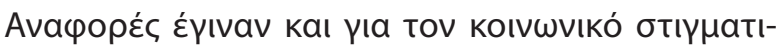

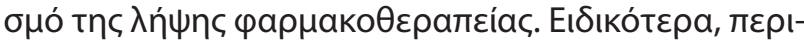
үра́

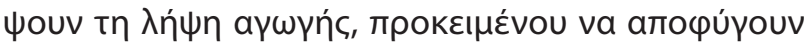

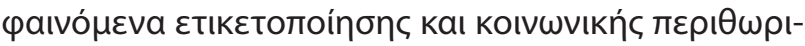
отоínonc.

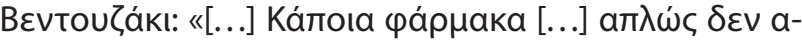

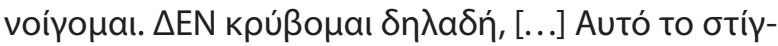

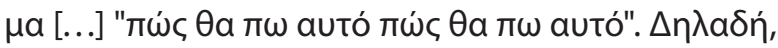

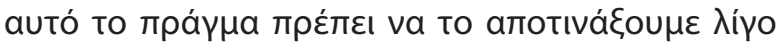

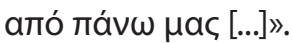

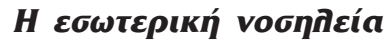

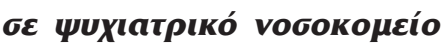

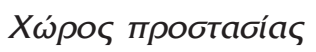

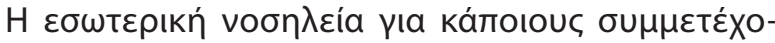

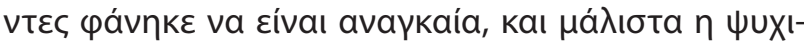

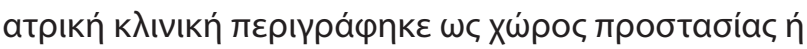

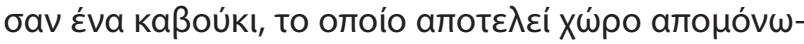

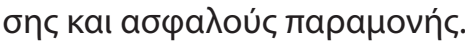

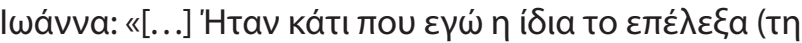

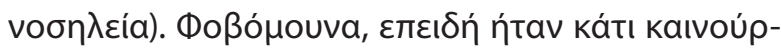

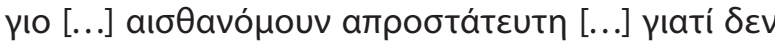

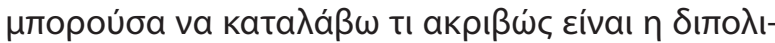

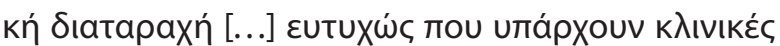

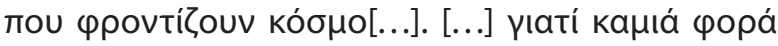
हívaı avaүkaío[...]».

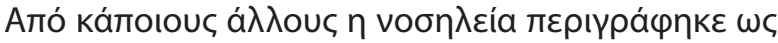

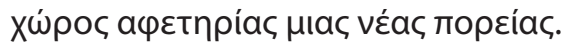

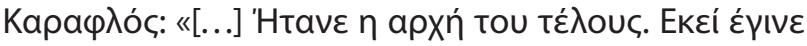

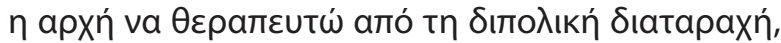

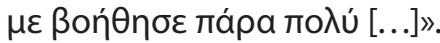




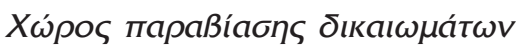

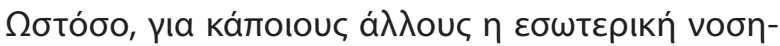

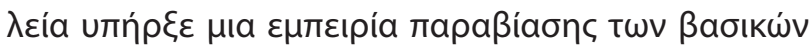

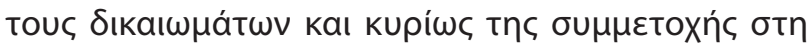

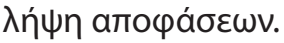

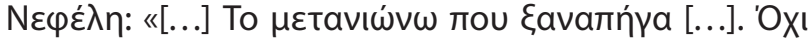

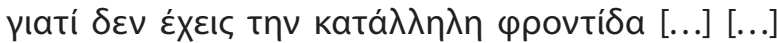

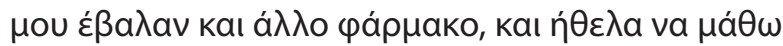

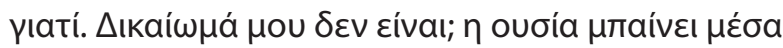

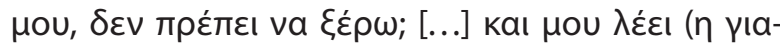

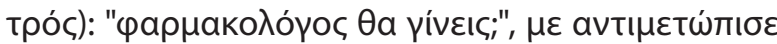

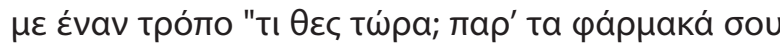

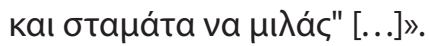

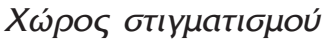

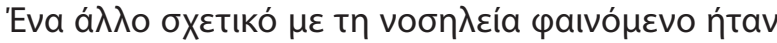

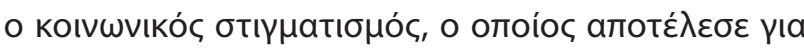

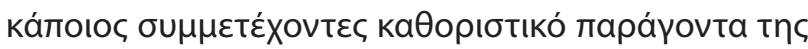

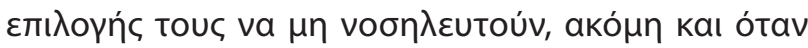

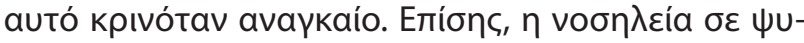

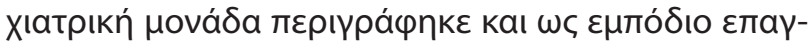

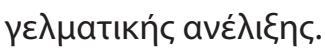

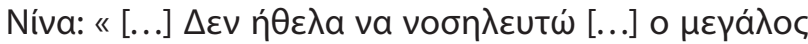

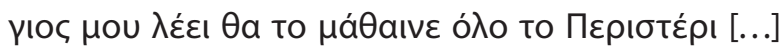

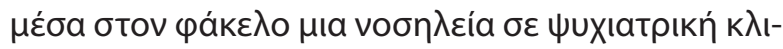

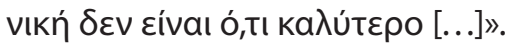

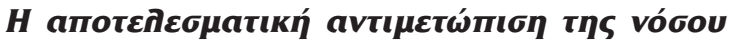

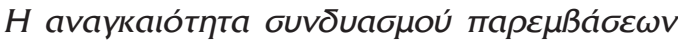

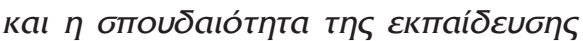

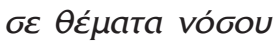

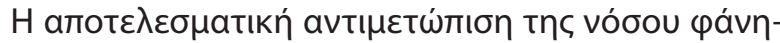

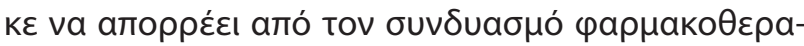

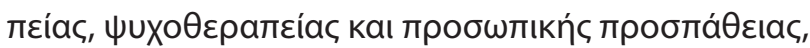

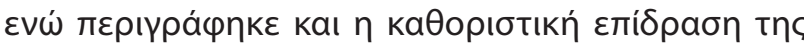

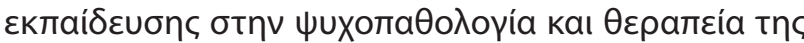
vóoou.

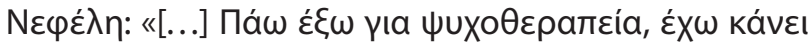

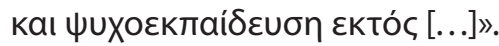

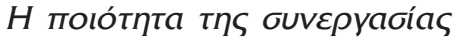

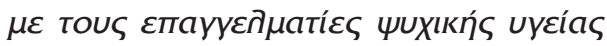

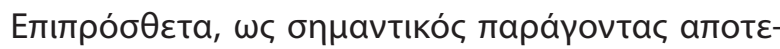

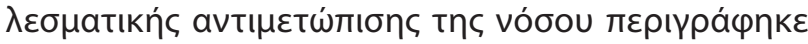

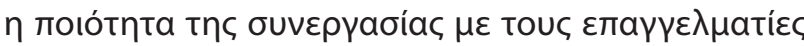
భuxıкńc uүzíac.

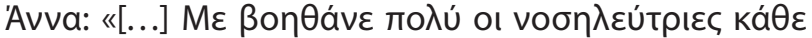

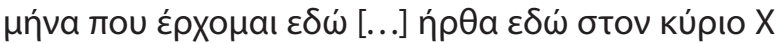

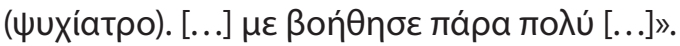

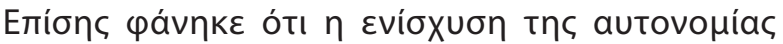

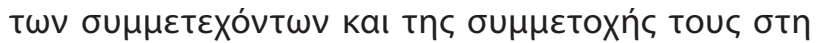

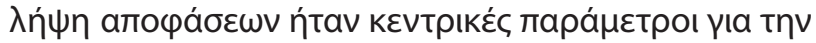

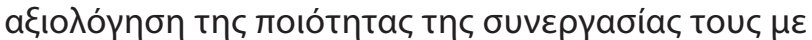

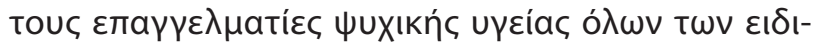
котท́т

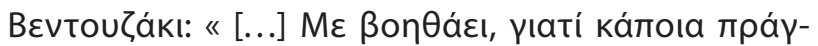

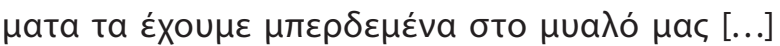

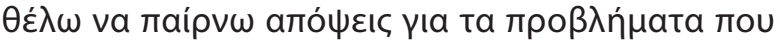

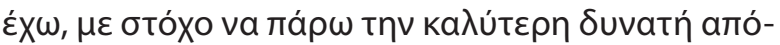

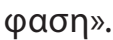

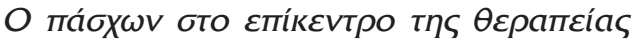

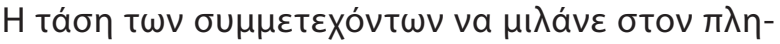

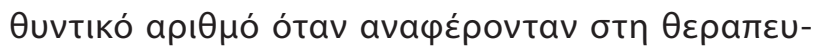

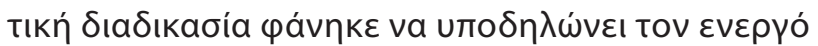

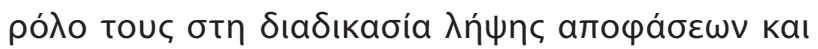

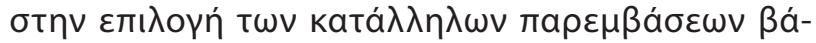

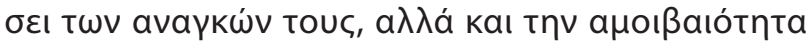

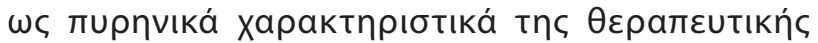

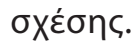

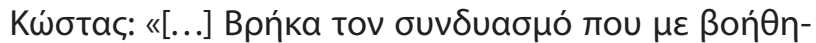

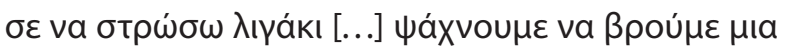

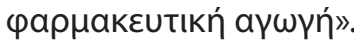

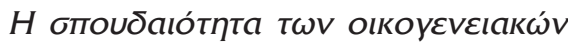

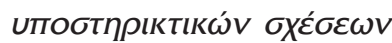

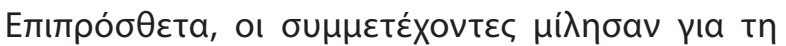

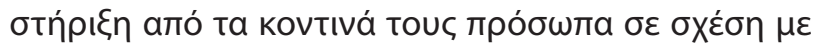

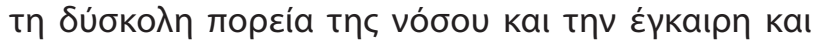

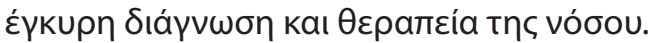

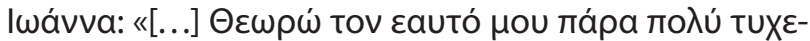

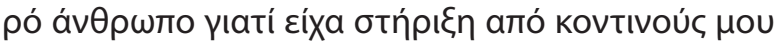

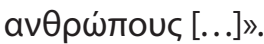

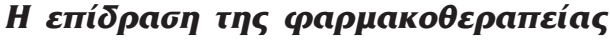

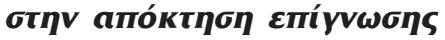

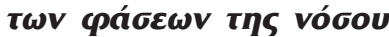

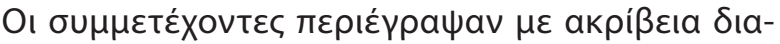

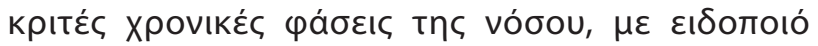

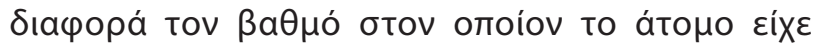

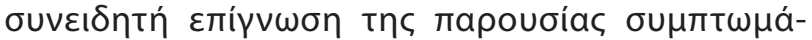

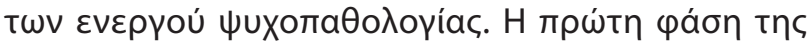




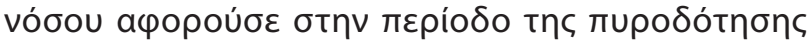

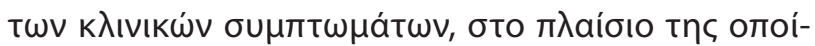

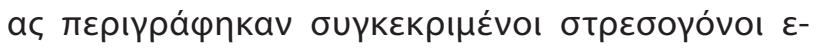

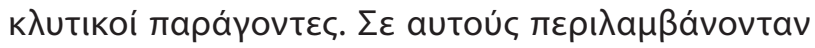

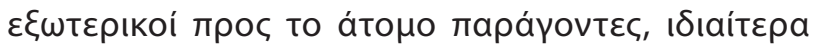

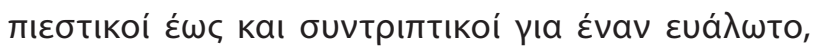

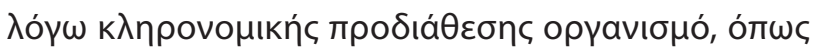

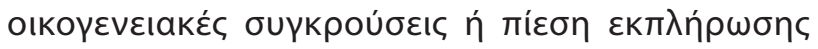

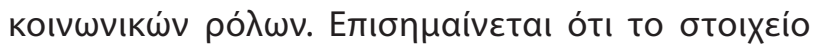

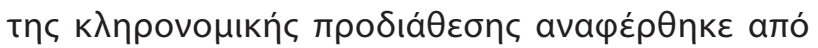

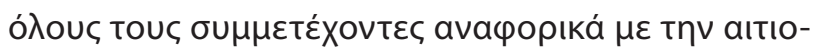

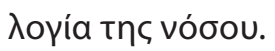

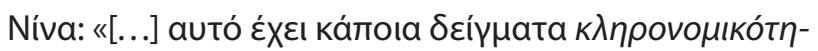

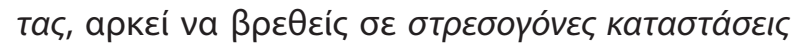
$[\ldots] »$.

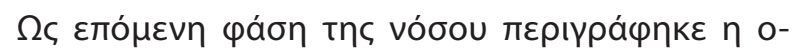

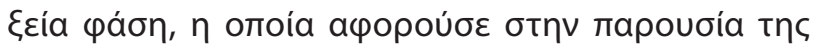

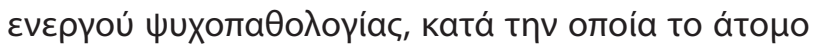

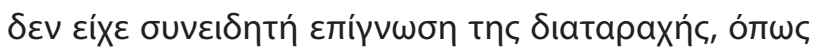

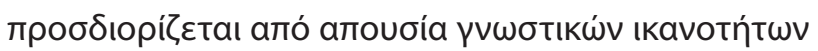

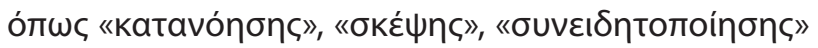

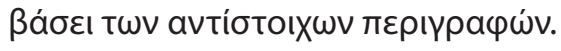

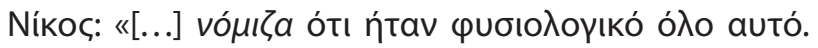

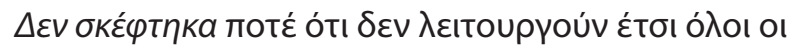

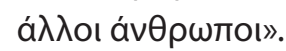

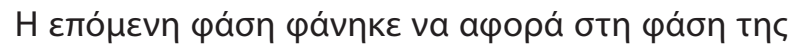

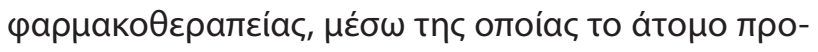

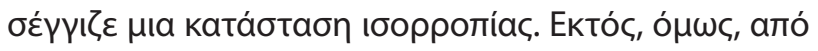

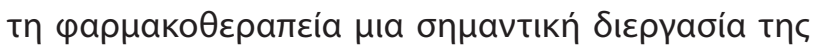

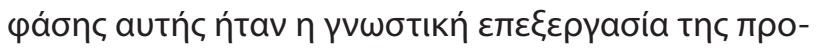

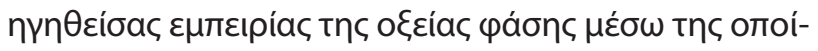
as to áto

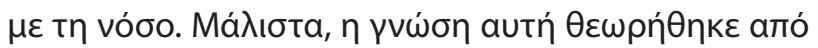

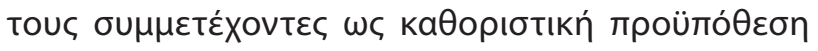

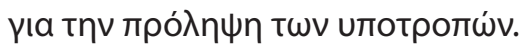

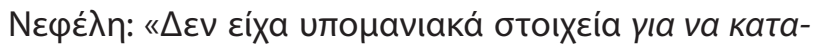

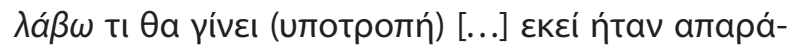

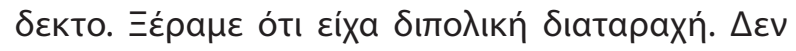

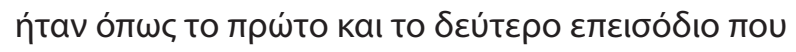
$\delta \varepsilon v \eta ́ \xi \varepsilon \rho a »$.

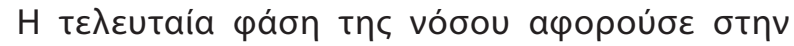

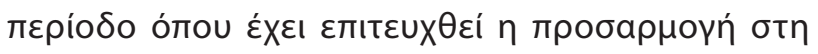

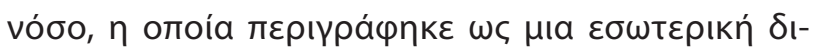

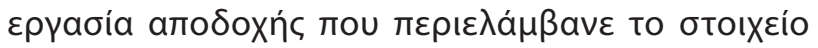

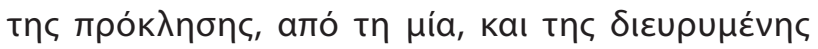

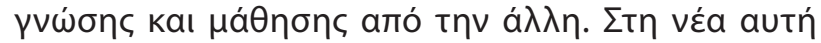

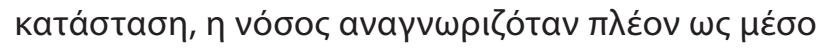

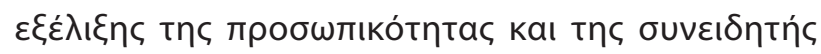
autenípvwonc.

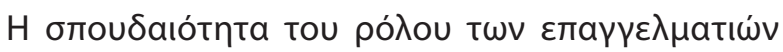

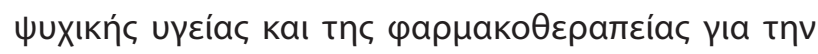

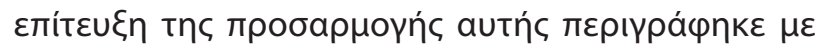

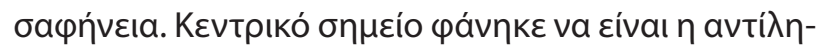

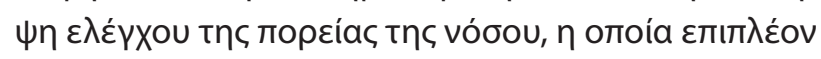

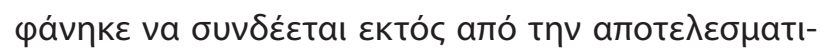

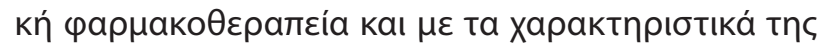

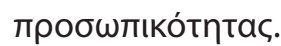

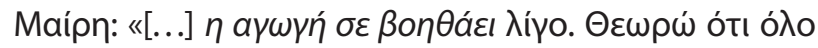

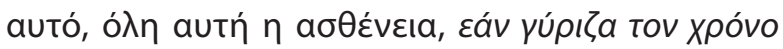

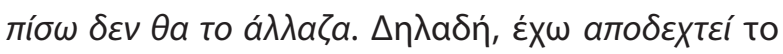

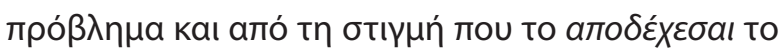

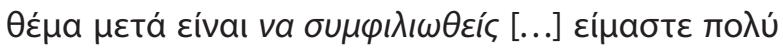

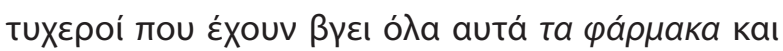

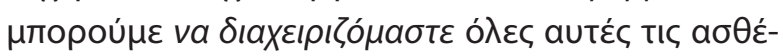

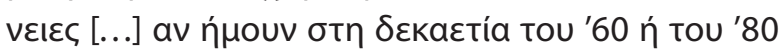

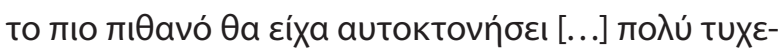

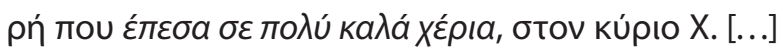
кaı $\theta a \mu a ́ \theta \omega$ va $\zeta \omega \mu \varepsilon$ autó».

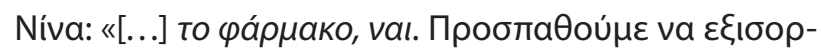

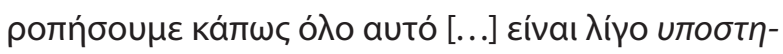

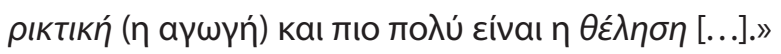

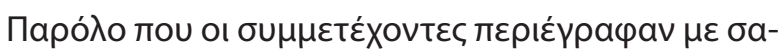

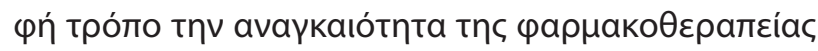

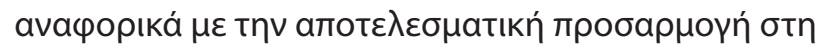

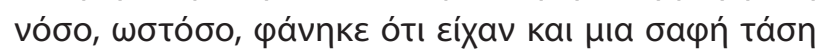

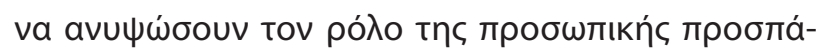

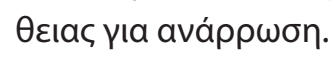

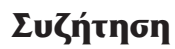

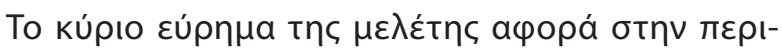

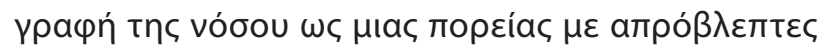

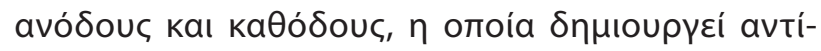

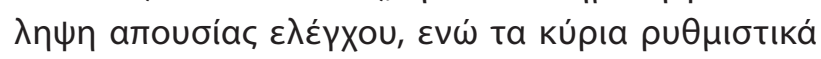

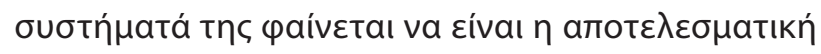

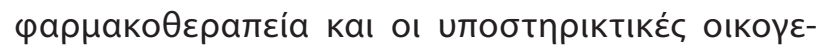

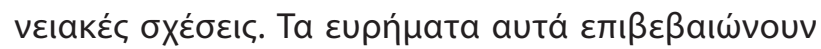

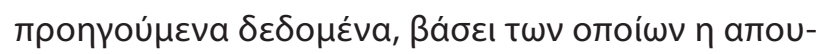

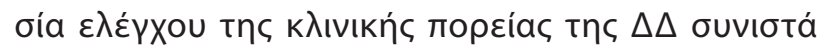

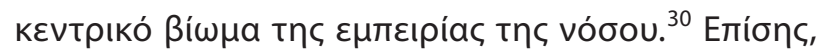

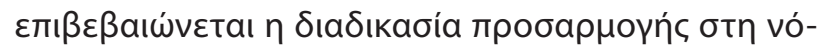




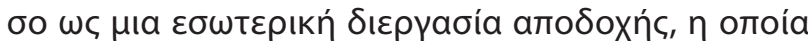

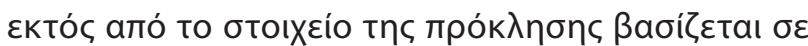

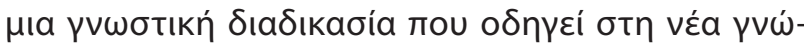

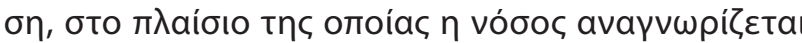

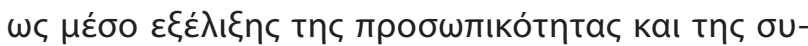

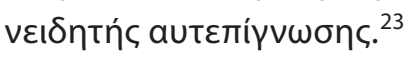

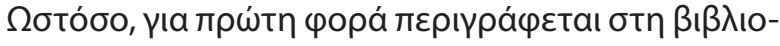

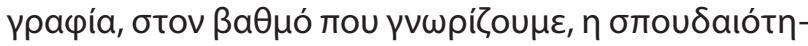

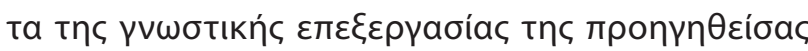

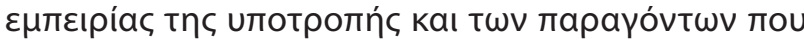

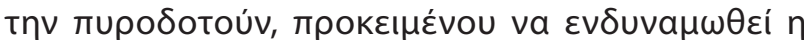

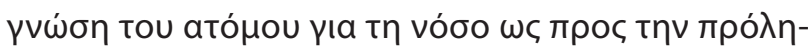

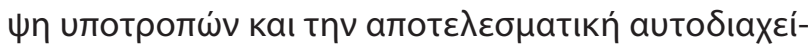

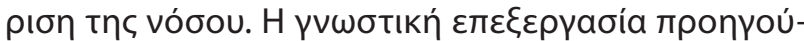

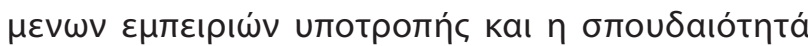

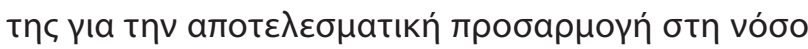

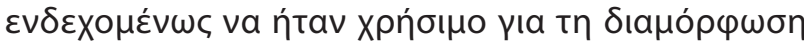

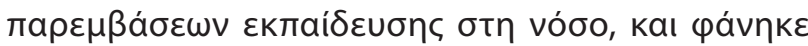

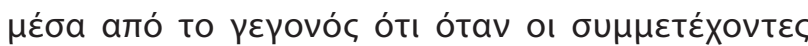

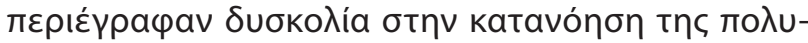

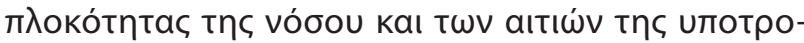

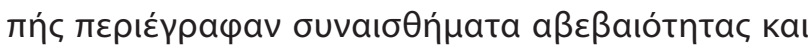
ह́vTaonc.

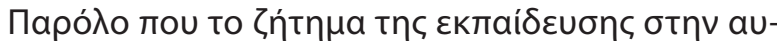

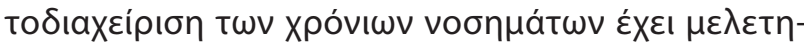

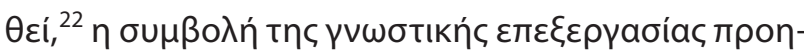

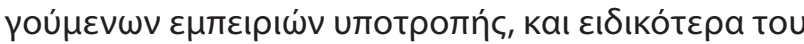

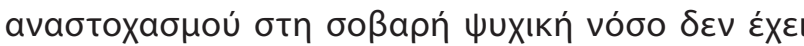

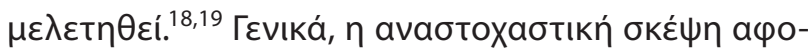

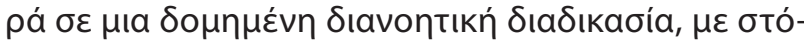

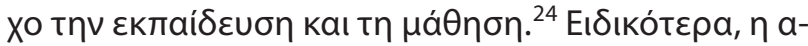

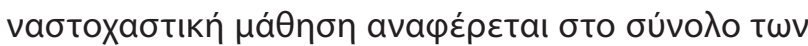

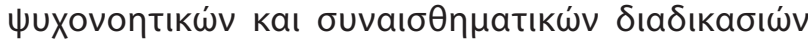

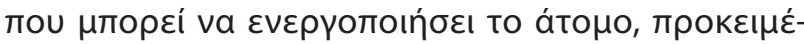
vou va ठı

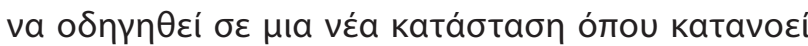

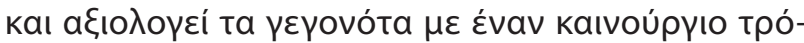

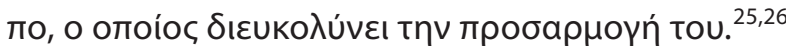

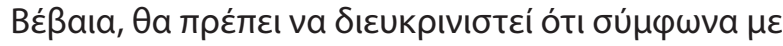

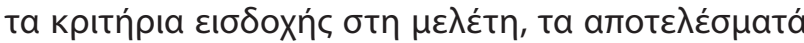

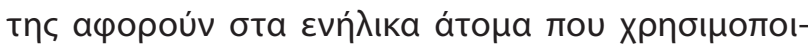

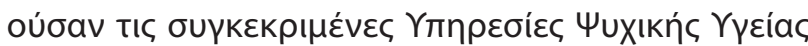

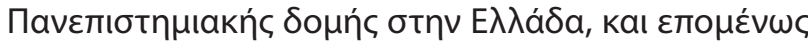

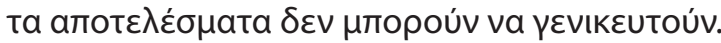

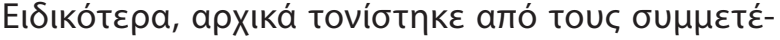

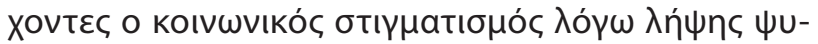

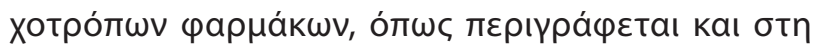

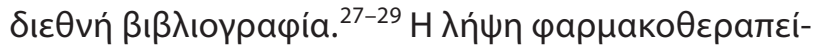

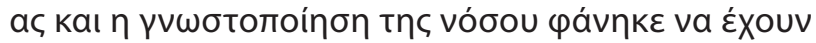

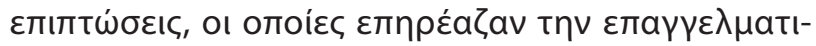

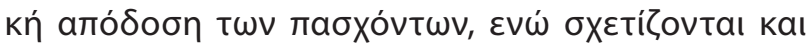

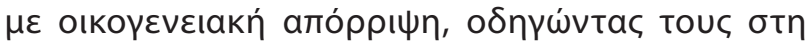

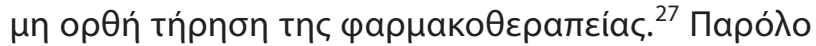

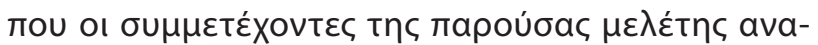

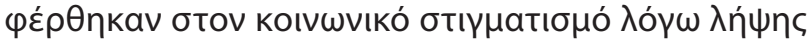

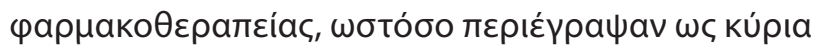

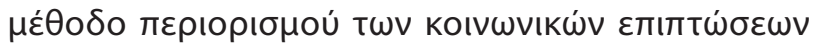

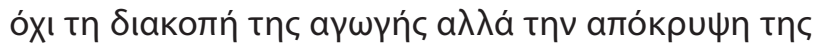

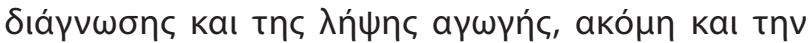

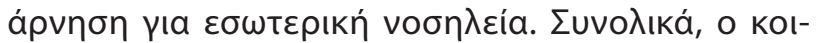

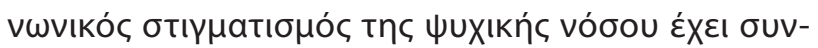

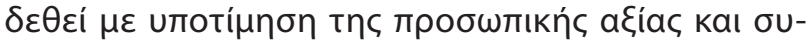

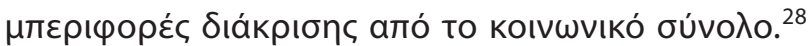

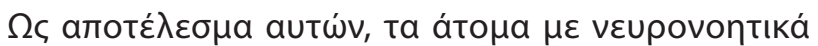

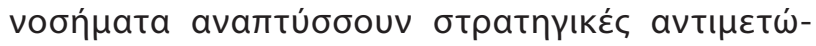

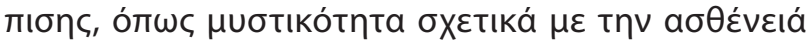

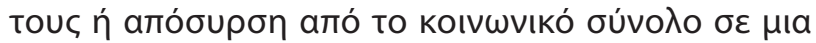

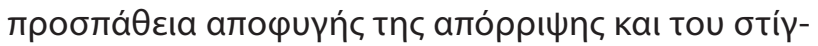

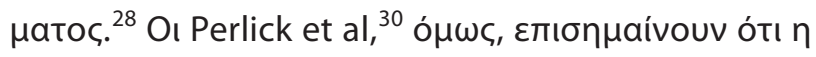

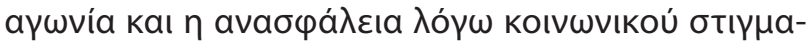

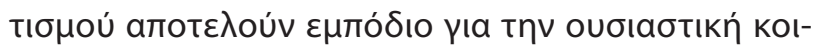

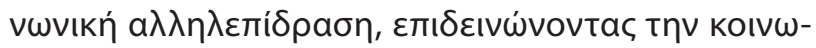

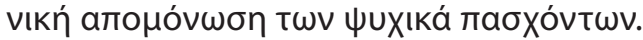

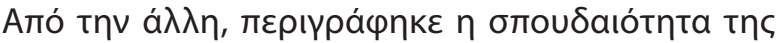

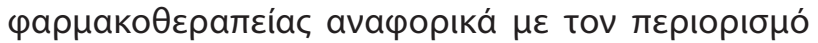

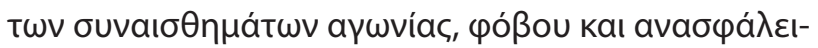

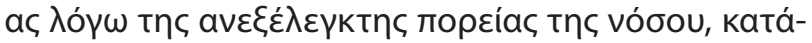

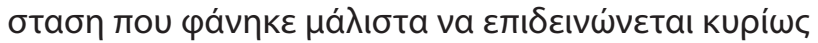
$\lambda$

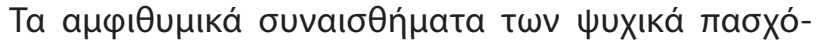

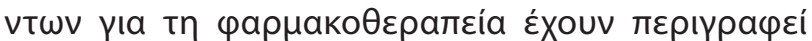

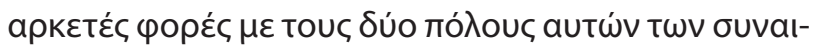

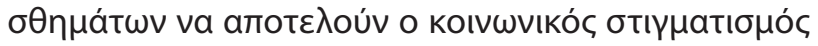

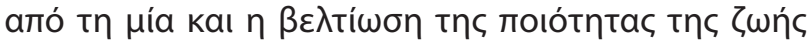

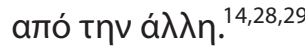

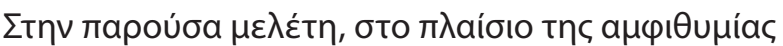

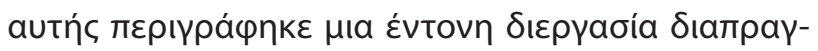

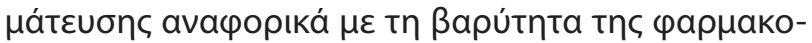

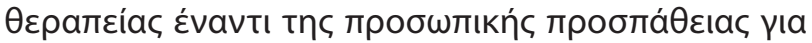




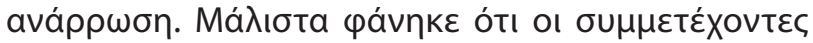

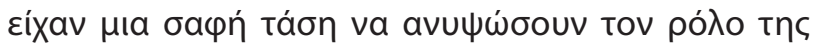

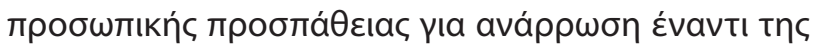

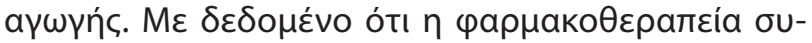

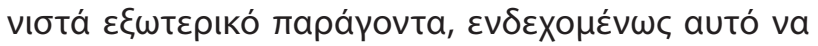

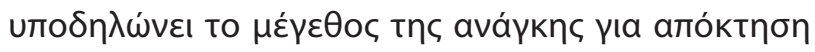

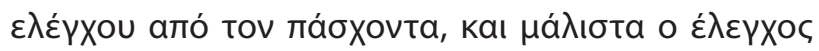

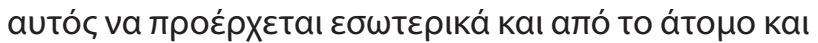

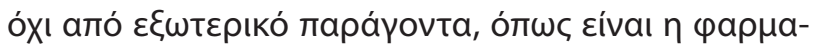

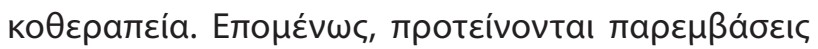

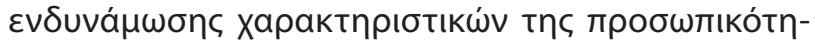

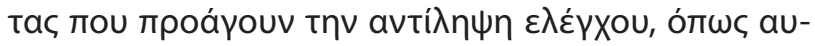

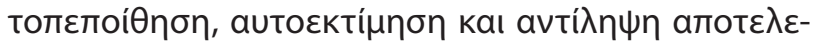

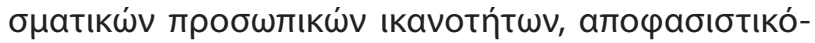

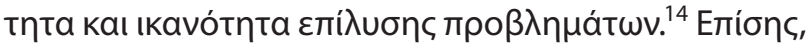

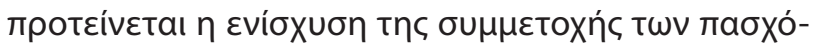

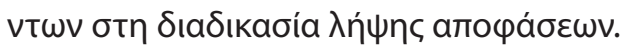

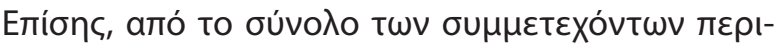

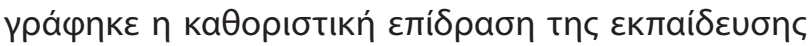

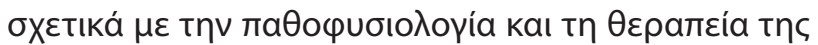

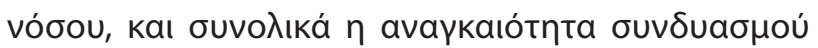

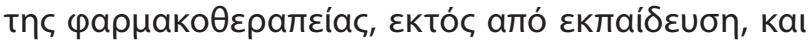

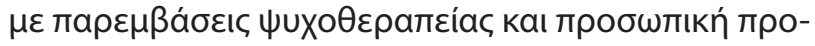

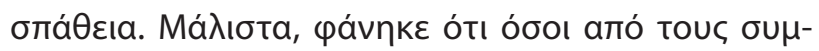

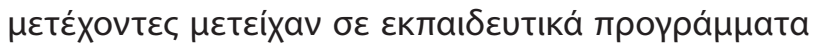

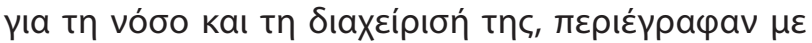

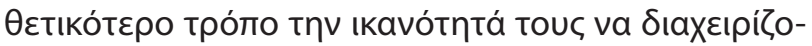

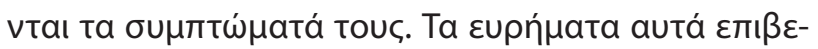

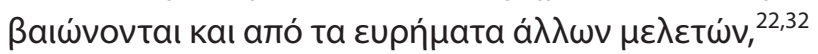

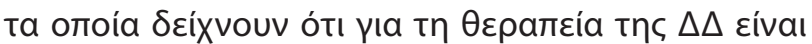

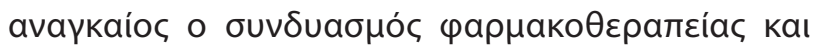

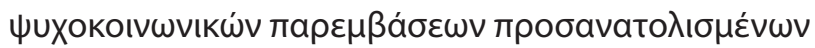

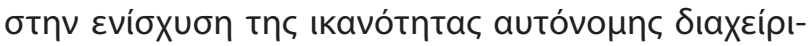

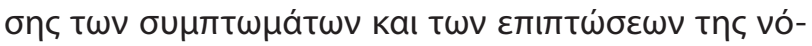

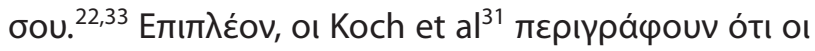

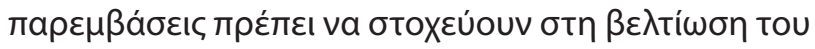

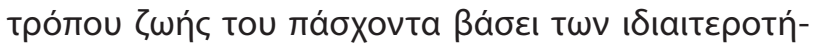

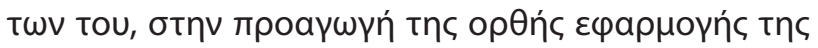

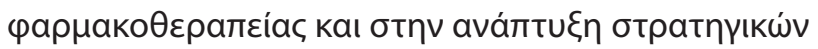

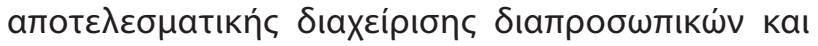

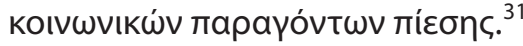

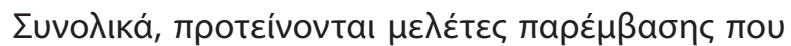

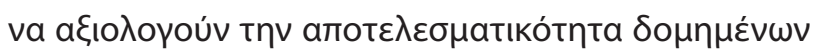

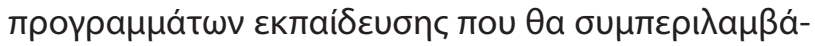

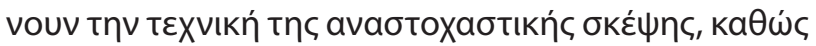

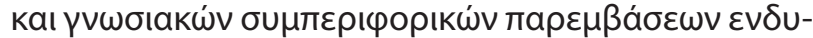

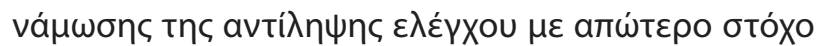

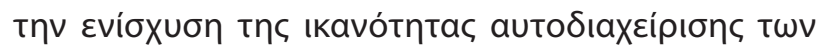

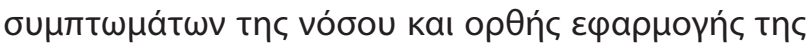
фарнакоӨврапві́ас.

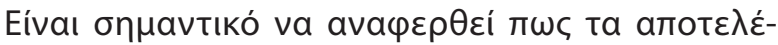

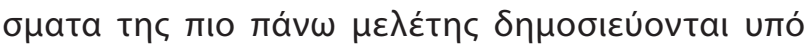

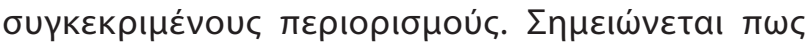

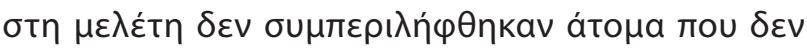

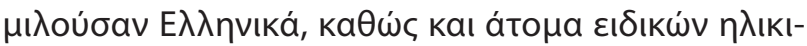

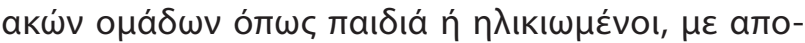

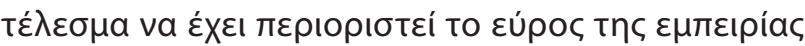

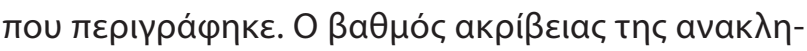

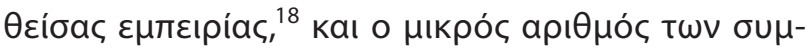

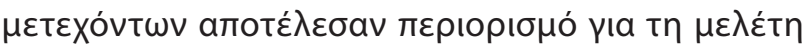

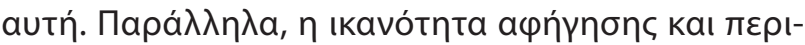

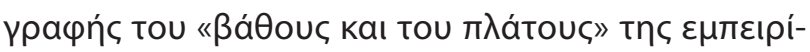

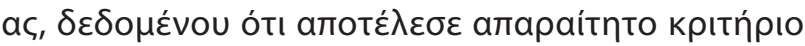

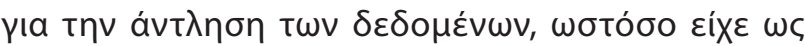

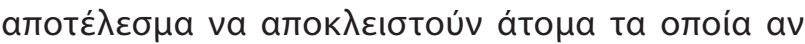

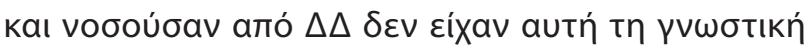

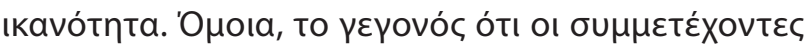

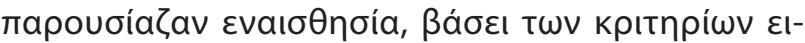

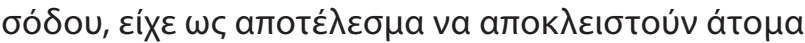

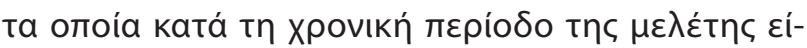

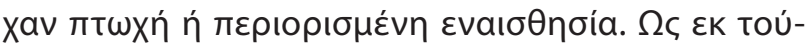

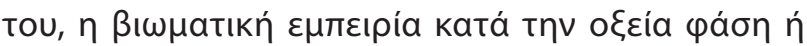

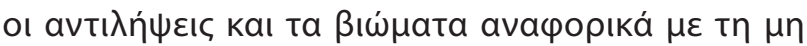

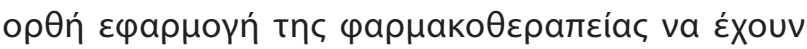

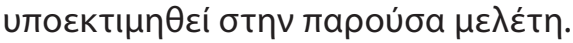

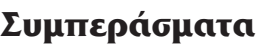

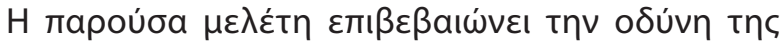

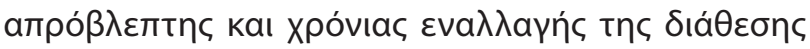
$\lambda$

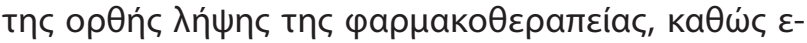

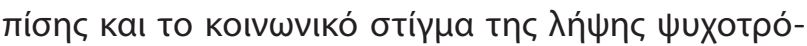

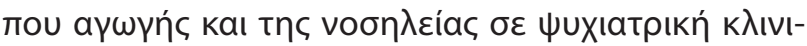

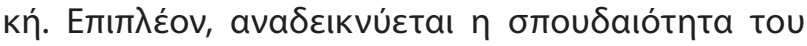

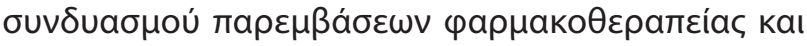

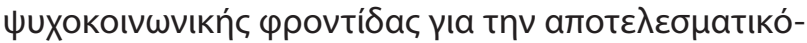

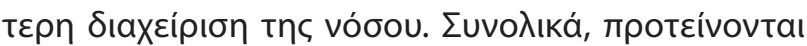

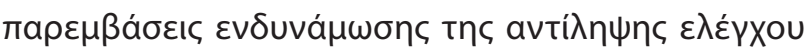

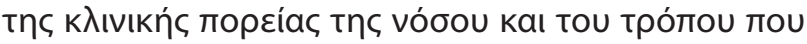

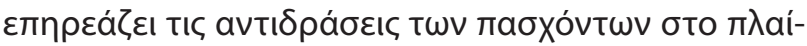

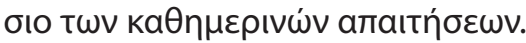




\title{
Perceptions regarding pharmacotherapy and effective treatment disease in people diagnosed with bipolar disorder
}

\author{
A. Hatzioannou, ${ }^{1,2}$ M. Karanikola, ${ }^{2}$ E. Papathanasoglou, ${ }^{3}$ \\ Aik. Kaikoushi, ${ }^{2,6}$ M. Nystazaki, ${ }^{4}$ G. Alevizopoulos ${ }^{4,5}$ \\ ${ }^{1}$ Cyprus Mental Health Services, Nicosia, Cyprus, \\ ${ }^{2}$ Department of Nursing, Cyprus University of Technology, Limassol, Cyprus, \\ ${ }^{3}$ Faculty of Nursing, University of Alberta, Edmonton, Alberta, Canada, \\ ${ }^{4}$ Department of Psychiatry, University of Athens, "Agioi Anargyroi" Hospital, Athens, \\ ${ }^{5}$ Department of Nursing, National and Kapodistrian University of Athens, Athens, Greece, \\ ${ }^{6}$ Mental Health Services, Famagusta, Cyprus
}

Psychiatriki 2017, 28:291-300

Bipolar disorder (BD) encompasses neuro-cognitive disturbances leading to psychological and social consequences affecting the quality of life of those suffering from it. However, the number of studies on the lived experience of people with $\mathrm{BD}$ about the treatment provided is relatively scant. The aim of this study is to investigate the lived experience of people with $B D$, focused on their perceptions and meanings about the treatment provided for their disorder. A qualitative, phenomenological design was applied. Following informed consent, thirteen people agreed to participate in the study, according to purposeful sampling and thematic saturation. Data collection was achieved through individual, semistructural interviews with open-ended questions, of a 30 minutes to an hour. The rigor of the analysis was validated according to Munhall's and van Manen's criteria. With regard to the main themes emerged, those revolved mainly around the social stigmatization following psychotropic medication. Further, the participants described anguish, fear and insecurity about the winding road nature of the disease, a condition that seemed to be worsened mainly due to discontinuation of medication, or alterations of the therapeutic schema. Interestingly, some of the participants described medication as a nutrient ingredient that kept them alive, thus revealing the importance they attached to psychotropic medication. Participants highlighted the importance of psychotropic medication, along with psychotherapy and personal effort, as well as education on topics related to psychopathology and treatment interventions for BD. Control over the clinical outcome of the disorder and self-management of the symptoms seem to be the ultimate need of people suffering from $\mathrm{BD}$, with a core association to the effective medication and psycho-education. Based on that, interventions aiming to patients' education in self-management skills are suggested. Furthermore, interventions towards the sensitization of the community on the biological aspects of mental disorders are proposed, tackling issues as stigma and medication concerns.

Key words: Mood disorders, lived experience, phenomenology, psychopharmacology, involuntary hospitalization, stigma, self-management.

\section{BıBลıорасpía}

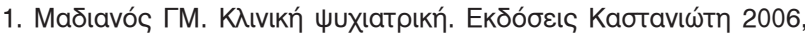
259-262, ISBN: 978-960-03-4243-7Top of Form Bottom of Form

2. Department of Health: Self care: a national view, 2007 Available from: http://webarchive.nationalarchives.gov.uk/20090120141703/ dh.gov.uk/en/healthcare/selfcare/index.htm
3. Colom F, Vieta E, Martinez-Aran A, Reinares M, Goikolea JM, Benabarre $A$ et al. A randomized trial on the efficacy of group psychoeducation in the prophylaxis of recurrences in bipolar patients whose disease is in remission. Arch Gen Psychiatry 2003, 60:402-407, doi:10.1001/archpsyc.60.4.402 
4. Thompson ML, Mental IIIness: Health and Medical Issues Today, 2006 Conn, Greenwood Press, ISBN-10: 0313335656, ISBN-13: 978-0313335655

5. Deegan PE. The Lived Experience of Using Psychiatric Medication in the Recovery Process and a Shared DecisionMaking Program to Support It. Psychiatr Rehabil J 2007, 31 62-69, PMID: 17694717

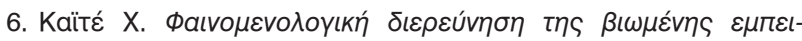

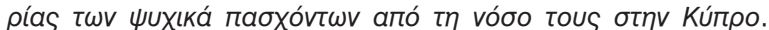

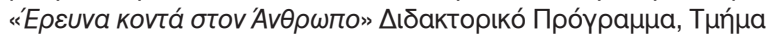

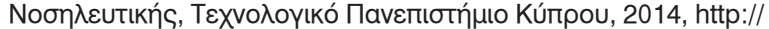
www.cut.ac.cy/digitalAssets/119/119054_booklet.pdf

7. RussellL, Moss D. Highandhappy? Exploring the experience of positive states of mind in people who have been given a diagnosis of bipolar disorder. Psychol Psychother 2012, 86:431-446, doi:10.1111/j.2044-8341.2012.02064.x

8. Warne T, Andrew S. "Re-searching for therapy: The ethics of using what we are skilled in". J Psychiatr Ment HIth Nurs 2010, 17503-509

9. Haddad PM, Brain C, Scott J. Non adherence with antipsychotic medication in schizophrenia: challenges and management strategies. Patient Relat Outcome Meas 2014, 23:43-62, doi: 10.2147/PROM.S42735

10. Piat M, Sabetti J, Couture A, Sylvestre J, Provencher H, Botschner $\mathrm{J}$ et al. What does recovery mean for me? Perspectives of Canadian mental health consumers. Psychiatr Rehabil 2009, 32:199-207, doi:10.2975/32.3.2009.199.207

11. Shattell MM, Starr S, Thomas SP. "Take my hand, help me out". Mental health service experience of the therapeutic relationship. Intern J Ment Hith Nurs 2007,16: 274-284, doi: 10.1111/j.14470349.2007.00477.x

12. Kaite $C$, Karanikola M, Merkouris A, Papathanassoglou EDE. "An Ongoing Struggle With the Self and Illness": A Meta-Synthesis of the Studies of the Lived Experience of Severe Mental IIIness. Arch Psychiatr Nurs 2015, 29:458-473

13. Henderson $\mathrm{C}$, Hales $\mathrm{H}$, Ruggeri M. Cross-cultural differences in the conceptualization of patients satisfaction with psychiatric services. Soc Psychiatry Psychiatr Epidemiol 2003, 38:142-148, doi: 10.1007/s00127-003-0606-7

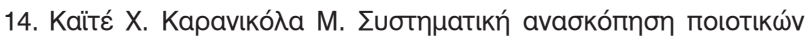

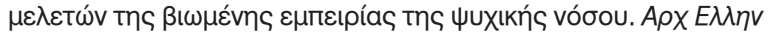
latp 2015, 32:451-460

15. George T. Defining care in the culture of the chronically mentally ill living in the community. J Transcult Nurs 2000, 11:102-110, doi: https://doi.org/10.1177/104365960001100204

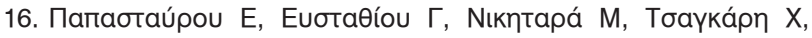

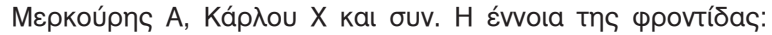

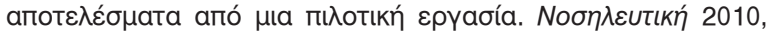
49:406-417

17. Panayiotopoulos C, Kerfoot, M. Evaluative Survey of Service Users and Professional Experiences of a Vocational Rehabilitation Unit for the Mentally III in Cyprus. Intern J Ment HIth 2013, 42:2-3

18. Munhall P. Revisioning Phenomenology. Nursing and Health Science Research. National League of Nursing, New York, 1994, http://trove.nla.gov.au/version/45499092

19. Ponty MM. The Primacy of Perception. Northwestern University Press 1964, http://www.hass.rpi.edu/public html/ruiz/Advanced IntegratedArts/ReadingsAIA/Merleau-Ponty_The\%20Primacy\%20 of\%20Perception.pdf

20. Manen MV. Researching Lived Experience: Human Science for an Action Sensitive Pedagogy. Series in the philosophy of education, 1990, SBN10: 0-7914-0425-0, ISBN13: 978-0-7914-0425-6

21. Burns N. Standards for qualitative research. Nurs Sci 1989, 2:44-52, doi: $10.1177 / 089431848900200112$
22. Crowe M, Inder D, Carlyle L, Wilson L, Whitehead A, Panckhurst $A$ et al. Feeling out of control: a qualitative analysis of the impact of bipolar disorder. J Psychiatr Ment Hith Nurs 2012, 19:294-302, doi: 10.1111/j.1365-2850.2011.01786.x

23. Hatfield AB, Lefley HP. Surviving Mental IIIness: Stress, Coping, and Adaptation. The Guilford Press 1993, Hash File: e9b7b97526fc375399468cff7d52b088.pdf

24. Lincoln TM, Wilhelm K, Nestoriu Y. Effectiveness of psychoeducation for relapse, symptoms, knowledge, adherence and functioning in psychotic disorders. Schizophr Research 2007, 96:232-245, doi:10.1016/j.schres.2007.07.022

25. Dewey J. How we think: A restatement of the relation of reflective thinking to the educational process. D.C. Heath and Company, 1933

26. Boud D. Reflection: Turning Experience into Learning Published in USA by Nichols Publication Data, 1985

27. Boyd EM, Fales AW. Reflective learning: the key to learning from experience. J Humanist Psychol 1983, 23:99-117

28. Lee $S$, Marcus $Y$, Chiub L, Tsangc A, Chuia $H$, Kleinmand $A$. Stigmatizing experience and structural discrimination associated with the treatment of schizophrenia in Hong Kong. Soc Sci Med 2006, 62:1685-1696

29. Link GB, Phelan, JC. Conceptualising stigma. Ann Rev Sociol 2001,27 363-385

30. Corrigan P, Wassel A. Understanding and influencing the stigma of mental illness. J Psychosoc Nurs Ment Hith Serv 2008, 46:42-48, PMID: 18251351

31. Perlick DA, Rosenheck RA, Clarkin JF, Sirey JA, Salahi J, Struening EL et al. Stigma as a barrier to recovery: adverse effects of perceived stigma on social adaptation of persons diagnosed with bipolar affective disorder. Psychiatr Serv 2001, 52:1627-1632, doi:10.1176/appi.ps.52.12.1627

32. Koch $\mathrm{T}$, Jenkin $\mathrm{P}$, Kralik $\mathrm{D}$ Chronic illness self-management: locating the 'self'. J Advanc Nurs 2004, 4:484-492, http://www. rima.org/web/medline_pdf/JAdvNurs_484-92.pdf

33. Candini V, Buizza C, Ferrari C, Caldera MT, Ermentini R, Ghilardi A et al. Is structured group psychoeducation for bipolar patients effective in ordinary mental health services? A controlled trial in Italy. J Affect Disord 2013, 151:149-155, doi: 10.1016/j. jad.2013.05.069

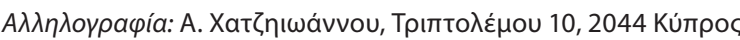
$\operatorname{T\eta \lambda }$ : (+357) 99896983

e-mail: Hatzioannou.a@hotmail.com 